\title{
Early Ultrastructural Changes in the Dorsal Skin Epidermis of W5istar-Derived Hypotrichotic WBN/ILA-Ht Rats after UVB-Irradiation
}

\author{
Valeria Malcotti ${ }^{1}$, Akira Yasoshima ${ }^{1}$, Koji Uetsuka ${ }^{1}$, Hiroyuki Nakayama ${ }^{1}$, and \\ Kunio Doi ${ }^{1}$ \\ ${ }^{1}$ Department of Veterinary Pathology, Faculty of Agriculture, The University of Tokyo, 1-1-1 Yayoi, Bunkyo-ku, Tokyo \\ 113-8657, Japan
}

\begin{abstract}
Early ultrastructural changes in the dorsal skin epidermis of Wistar-derived hypotrichotic WBN/ILA- $H t$ rats were examined for up to 12 hours after UVB-irradiation $\left(10 \mathrm{~kJ} / \mathrm{m}^{2}\right)(12 \mathrm{HAI})$. The first change appeared at $3 \mathrm{HAI}$ as swelling and/or vacuolation of mitochondria in the spinous and basal cells. The number of keratinocytes with such mitochondrial changes decreased thereafter. At 6 HAI, apoptosis of basal cells developed. These cells showed shrinkage of cell bodies with condensed or fragmented nuclei and dark cytoplasm due to densely packed bundles of tonofilaments. At the same time, keratinocytes with prominent nucleoli were also observed in the basal and suprabasal layers. At 12 HAI, the number of keratinocytes with prominent nucleoli increased, and mild and focal intercellular edema developed mainly in the basal layer. In addition, a small number of apoptotic basal cells were still observed. The present ultrastructural findings supported the histopathological findings previously reported by our research group. (J Toxicol Pathol 2001; 14: 173-177)
\end{abstract}

Key words: dorsal skin epidermis, WBN/ILA- $H t$ rat, UVB-irradiation, ultrastructure

The UV-induced dermatoses and tumors are said to have a close relation with the depletion of the ozone layer and a consequent increase in the intensity of ultraviolet (UV) radiation reaching the earth's surface. Among UVA (320$400 \mathrm{~nm}), \mathrm{UVB}(290-320 \mathrm{~nm})$ and UVC (200-290 nm), UVB is called the sunburn spectrum or the erythemal band because it causes sunburn reaction with erythema in human skin $^{1}$. Absorption of UVB occurs primarily in the epidermis ${ }^{2}$, causing molecular damage to nucleic $\mathrm{acid}^{3}$ and proteins ${ }^{4,5}$.

The cellular damage in the skin caused by UVBirradiation is histopathologically characterized by formation of so-called "sunburn" cells in the basal and suprabasal layers of the epidermis ${ }^{6,7}$. Under light microscope, sunburn cells show eosinophilic cytoplasm with or without shrunken and condensed nucleus which is strongly stained with terminal deoxy nucleotidyl transferase (TdT)-mediated dUTP-digoxigenin nick end labeling (TUNEL) method for the in situ detection of fragmented DNA ${ }^{8}$. These cells are considered as a cell population of keratinocytes undergoing

Received: 20 April 2001, Accepted: 8 May 2001

Mailing address: Valeria Malcotti, Department of Veterinary

Pathology, Faculty of Agriculture, The University of Tokyo, 1-1-1

Yayoi, Bunkyo-ku, Tokyo 113-8657, Japan

TEL/FAX: 81-3-5841-8185

E-mail: aa87181@mail.ecc.u-tokyo.ac.jp apoptosis ${ }^{9}$, and the induction of apoptosis by UVB irradiation has been considered to play a physiologically important role in eliminating DNA-damaged cells from the skin $^{10 .}$

The aim of this study is to clarify the ultrastructural characteristics of UVB-induced early lesions in the dorsal skin epidermis of WBN/ILA- $H t$ rats (HtRs). HtR has an autosomal dominant gene ( $H t$ : dominant hypotrichosis) responsible for the characteristics of hypotrichosis ${ }^{11}$ and is considered to be a useful laboratory animal for dermatotoxicity study ${ }^{12-15}$.

Six 7-week-old HtRs (Saitama Experimental Animal Supply Co., Saitama) were used. They were individually kept in isolator cages (Niki Shoji Co., Ltd., Tokyo) in an animal room under controlled conditions (temperature, $23 \pm$ $2^{\circ} \mathrm{C}$; relative humidity, $55 \pm 5 \%$ ) and fed pelleted diet, MF (Oriental Yeast Co., Tokyo) and tap water ad libitum throughout the experimental period.

The dorsal skin of 4 animals was irradiated with artificial UVB light (wavelength: $290-320 \mathrm{~nm}$ ) (ATTO Co., Tokyo) for 70 minutes at $10 \mathrm{~cm}$ below the light source (irradiation dose: $10 \mathrm{~kJ} / \mathrm{m}^{2}$ ). The dose and exposure time were decided based on the results of the previous studies ${ }^{8,16}$. One skin sample/animal was obtained from 4 irradiated animals with a $6 \mathrm{~mm}$ biopsy punch (Nagatoishi Co., Tokyo) under ether anesthesia at 1, 3, 6 and 12 hours after the 
irradiation (HAI), respectively. In addition, one skin sample/animal was obtained from 2 unirradiated control animals in the same way.

Small pieces of the skin samples were fixed in $2.5 \%$ glutaraldehyde in $0.1 \mathrm{M}$ phosphate buffer ( $\mathrm{pH} 7.4)$, postfixed in $1 \%$ osmium tetroxide in the same buffer, and embedded in EPOK 812 (Ohken Co., Ltd., Tokyo). Ultrathin sections were double-stained with uranyl acetate and lead citrate and observed under a JEM-100EX electron microscopy (JEOL Co., Ltd., Tokyo).

At $1 \mathrm{HAI}$, ultrastructural features of the dorsal skin in the UVB-group were similar to those in the control one (Figs. 1a and b). At 3 HAI, swelling and/or vacuolation of mitochondria developed in keratinocytes, and these ultrastructural changes were considered to correspond to intracytoplasmic edema of keratinocytes observed under light microscope ${ }^{8,16}$. Such mitochondrial changes were generally seen in keratinocytes in the spinous layer, but they sometimes extended to keratinocyets in the basal layer (Fig. $2)$. The number of keratinocytes with mitochondrial swelling and/or vacuolation decreased thereafter.

The most prominent ultrastructural change at $6 \mathrm{HAI}$
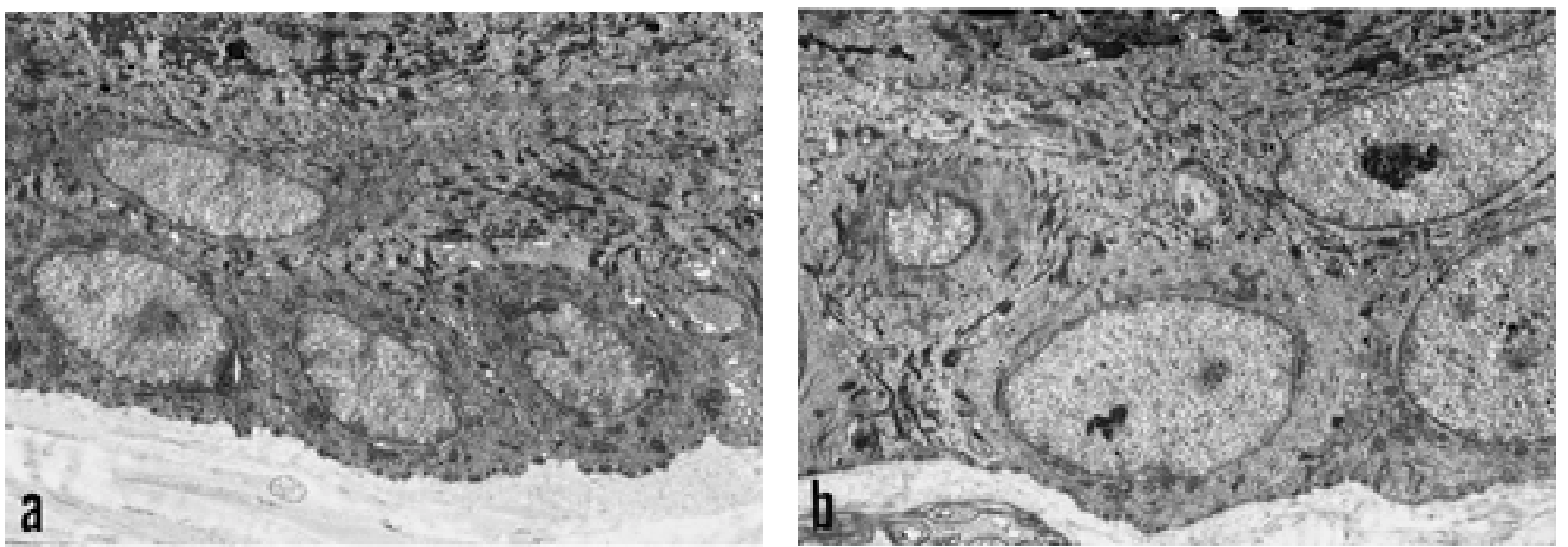

Fig. 1. Dorsal skin epidermis of a control rat (a) and a UVB-irradiated rat at $1 \mathrm{HAI}(\mathrm{b})$. There is no difference between (a) and (b). $\times 3,000$.

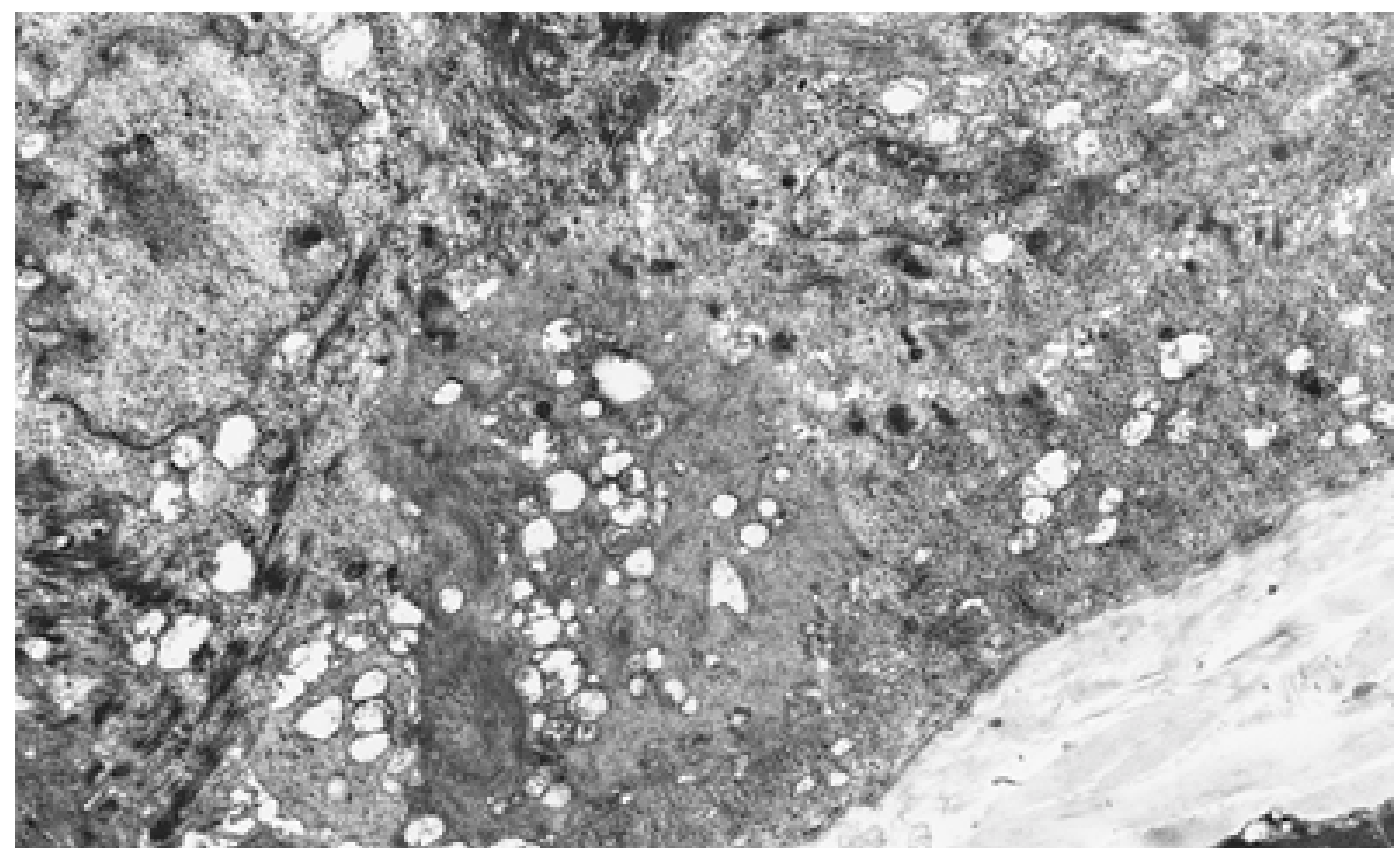

Fig. 2. Dorsal skin epidermis of a UVB-irradiated rat at 3 HAI. Prominent mitochondrial swelling and vacuolation in spinous and basal cells. $\times 6,250$. 


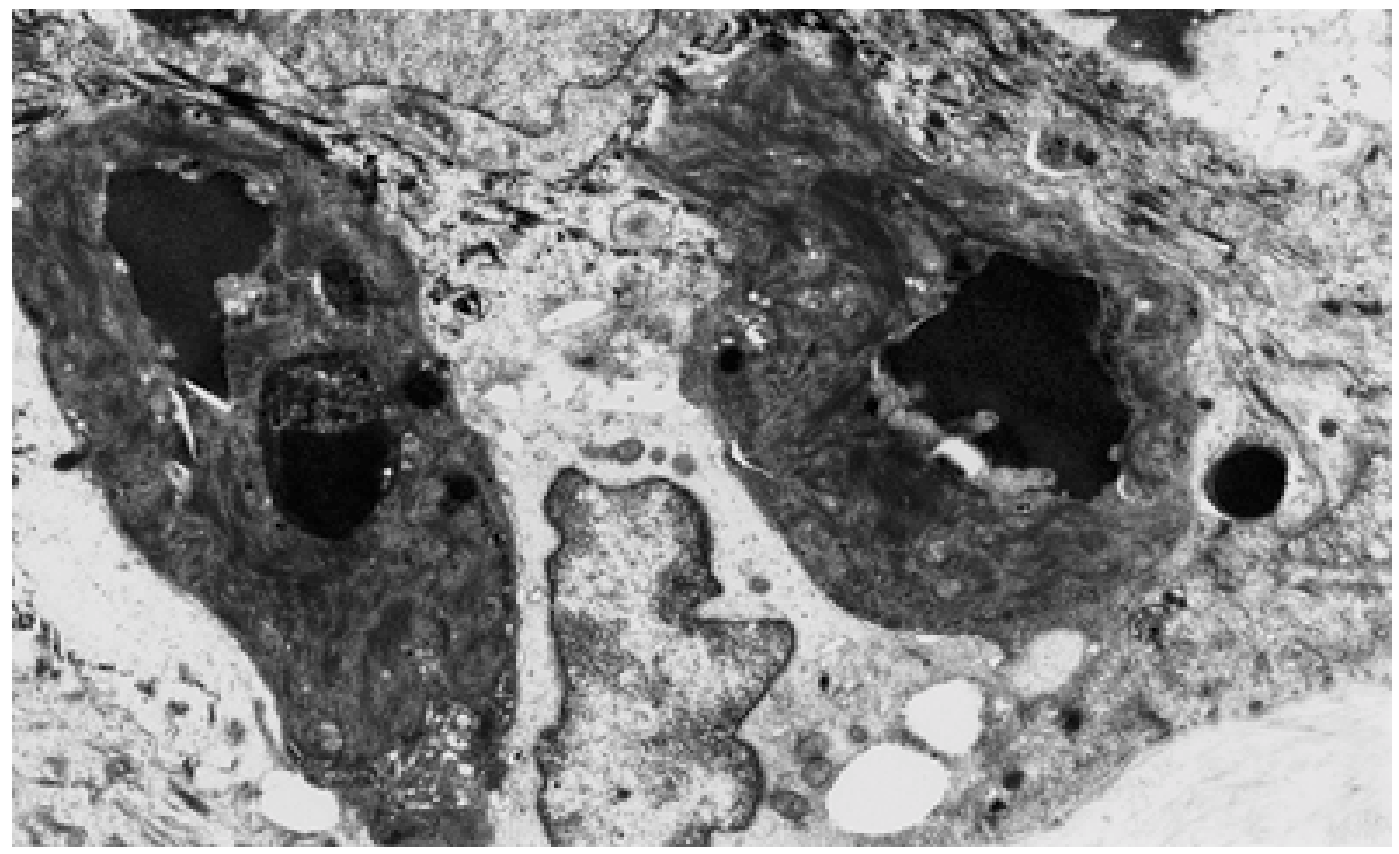

Fig. 3. Basal cells in the dorsal skin epidermis of a UVB-irradiated rat at 6 HAI. Apoptotic cells characterized by condensed and/or fragmented nucleus and dark cytoplasm due to densely packed tonofilament bundles. $\times 6,250$.

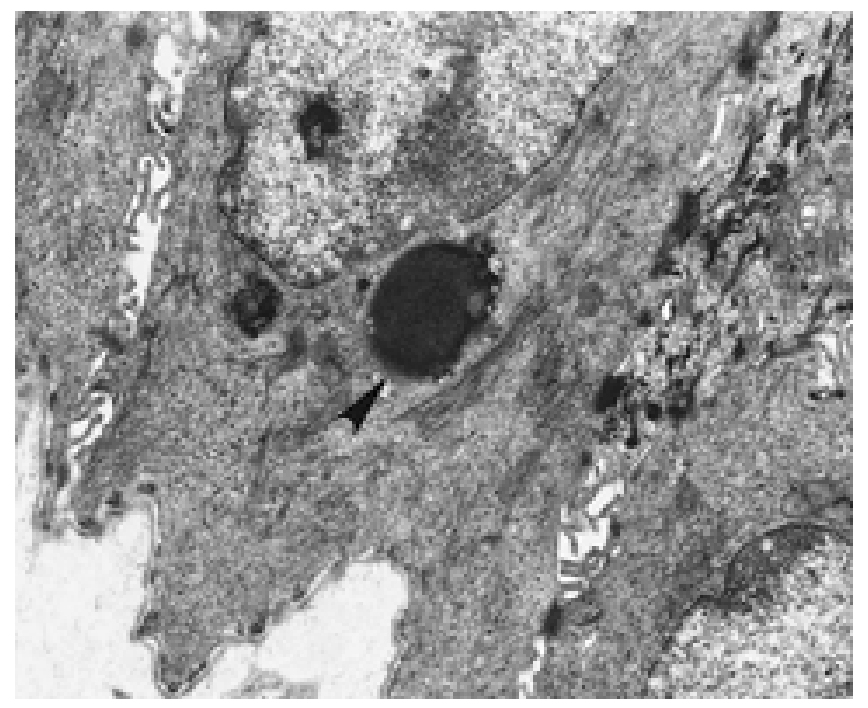

Fig. 4. Basal cells in the dorsal skin epidermis of a UVB-irradiated rat at 12 HAI. An apoptotic body (arrowhead) phagocytized by a keratinocyte. $\times 8,000$.

was an appearance of apoptotic basal cells. These cells unexceptionally decreased in their size and were generally characterized by marked condensation of nuclear chromatin and/or nuclear fragmentation as well as by dark cytoplasm due to densely packed bundles of tonofilaments (Fig. 3). The basal cells with these ultrastructural characteristics were thought to be sunburn cells observed under light microscope $e^{8,9,16}$. Remnants of apoptotic cells or apoptotic bodies were removed later by phagocytosis by adjacent keratinocytes (Fig. 4). Interestingly, keratinocytes with prominent nucleoli were simultaneously observed in the basal and suprabasal layers at 6 HAI (Fig. 5), and this suggests the initiation of cell proliferation. In this connection, it is reported that UVB also activates a proliferative pathway to replace apoptotic cells ${ }^{17}$.

At $12 \mathrm{HAI}$, the number of keratinocytes with prominent nucleoli increased, and mild and focal intercellular edema developed mainly in the basal layer (Fig. 6). In addition, apoptotic cells were still observed, though their number decreased.

In conclusion, the early development of ultrastructural changes in the dorsal skin epidermis of rats after UVBirradiation was clarified in the present study, and these ultrastructural findings supported the light microscopical ones previously reported by our research group.

\section{References}

1. Benjamin SA. Radiation and Heat. In: Hanbook of Toxicologic Pathology, Haschek WM, Rousseaux CG (eds), 984-1045, 1991.

2. Anderson RR. The optics of human skin. J Invest Dermatol 1981; 77: 13-19.

3. Brash DE, Rudolph JA, Simon JA, Lin A, McKenna GJ, Baden HP, Halperin AJ, and Pontén J. A role for sunlight in skin cancer: UV-induced p53 mutations in squamous cell carcinoma. Proc Natl Acad Sci USA 1991; 88: 1012410128.

4. Zamansky GB. Environmental wavelengths of ultraviolet 


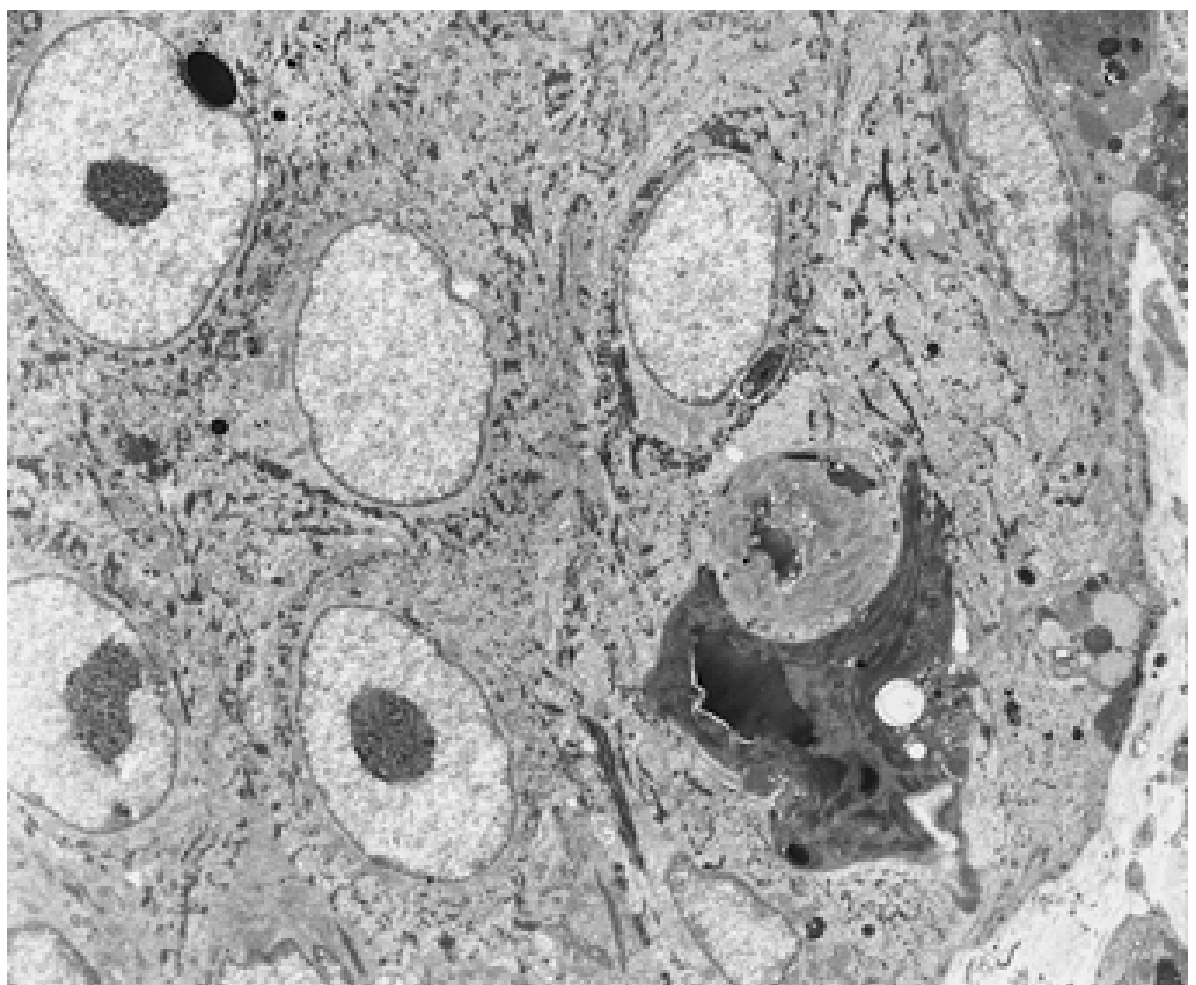

Fig. 5. Dorsal skin epidermis of a UVB-irradiated rat at 6 HAI. Many keratinocytes with prominent nucleolus and an apoptotic basal cells are simultaneously seen. $\times 3,000$.

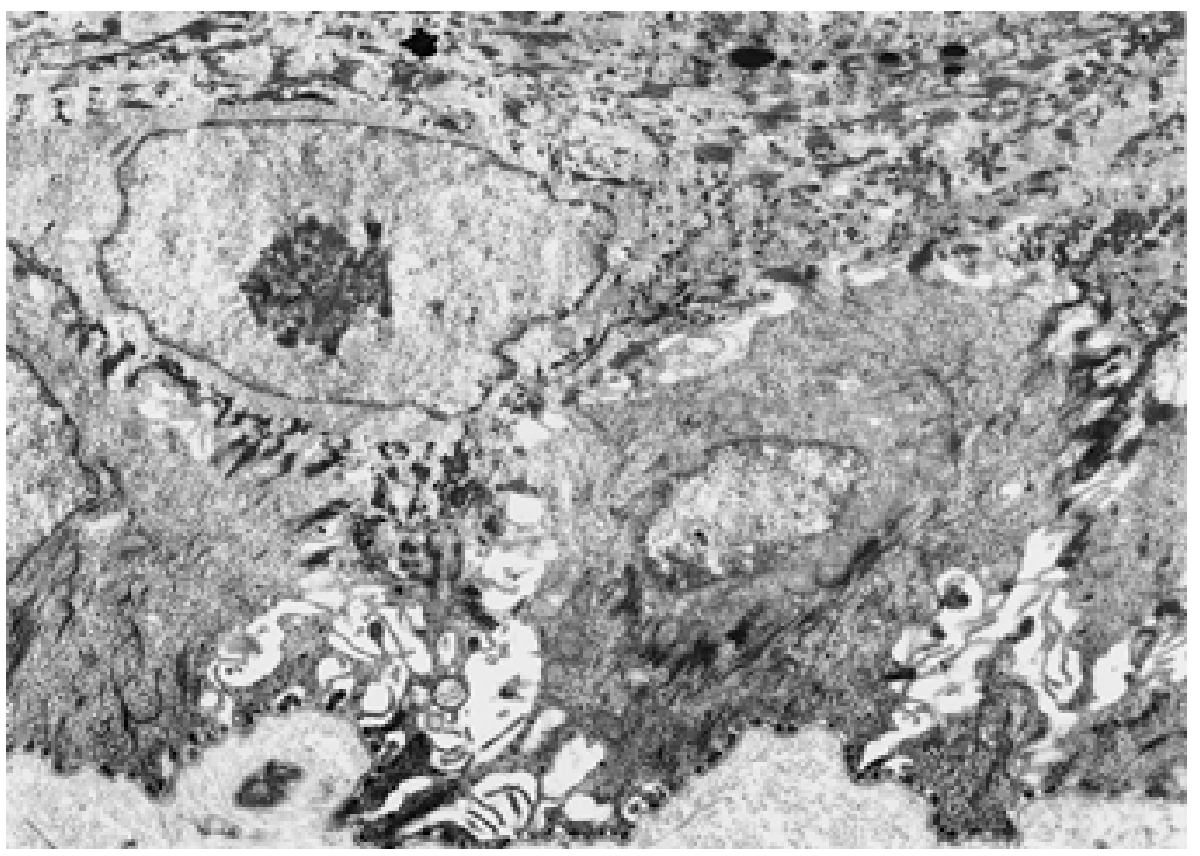

Fig. 6. Dorsal skin epidermis of a UVB-irradiated rat at 12 HAI. Mild and focal intercellular edema is seen in the basal layer. $\times 6,250$. 
light induce cytoskeletal damage. J Invest Dermatol 1987; 89(6): 603-606.

5. Devary Y, DiDonato JA, and Karin M. NF-KB activation by ultraviolet light not dependent on a nuclear signal. Science 1983; 261: 1442-1445.

6. Young A. The sunburn cell. Photodermatology 1987; 4: 127-134.

7. Haak AR. Cell death by apoptosis in epidermal biology. J Invest Dermatol 1993; 101: 107-112.

8. Kuroki K, Kimura T, Nakayama H, and Doi K. Dorsal skin responses to UVB-irradiation in Wistar-derived hypotrichotic WBN/ILA-Ht rats (HtRs). Exp Toxicol Pathol 2001; (in press).

9. Kane K. Ultraviolet B-induced Apoptosis of keratinocytes in murine skin is reduced by mild local hyperthermia. J Invest Dermatol 1995; 104(1): 62-67.

10. Lane DP. A death in the life of p53. Nature 1993; 362: 786787.

11. Nishimura M. Genetics of dominants hairless rats. Lab Anim 1987; 4: 13-14.

12. Iwamoto $\mathrm{S}$, Nakayama $\mathrm{H}$, Yasoshima $\mathrm{A}$, and Doi $\mathrm{K}$. Hydrogen peroxide-induced dermatitis in WBN/ILA-Ht rats.
Exp Anim 1997; 46: 147-152.

13. Iwamoto $S$, Nakayama $H$, and Doi K. Morphological and morphometrical study on the dorsal skin of Wistar and WBN/ILA-Ht rats in their developing stage. Evaluation of the proliferation and apoptotic processes. Histol Histopathol 1998; 13: 981-988.

14. Kuroki K, Kimura T, Nakayama H, and Doi K. Manganese ethylene bis (Maneb)-induced degeneration of hair follicle epithelia in the dorsal skin of WBN/ILA-Ht rats. J Toxicol Pathol 1998; 11: 205-207.

15. Albarenque SM, Shinozuka J, Iwamoto S, Nakayama $H$, and Doi K. T-2 toxin-induced acute skin lesions in Wistarderived hypotrichotic WBN/ILA-Ht rats. Histol Histopathol 1999; 14: 337-342.

16. Malcotti V, Kuroki K, Nakayama H, and Doi K. Effects of double UVB-irradiations with different intervals on the dorsal skin of Wistar-derived hypotrichotic WBN/ILA- $H t$ rats. Exp Toxicol Pathol 2001; (in press).

17. Ouhtit A, Davis DW, Ullrich SE, McConkey D, and Ananthaswamy HN. Temporal events in skin injury and the early adaptive responses in Ultraviolet-irradiated mouse skin. Am J Pathol 2000; 156(1): 201-207. 